\title{
Structure, colours, and populations of NGC 3115
}

\section{Mysteries of a well-known object}

\author{
R. Michard \\ Observatoire de Paris, LERMA, 77 av. Denfert-Rochereau, 75015 Paris, France \\ e-mail: raymond.michard@obspm.fr \\ Received 7 September 2006 / Accepted 21 November 2006 \\ ABSTRACT

\begin{abstract}
Visible and near IR colour distributions in NGC 3115 have been studied from a set of frames of the Las Campanas Observatory and the 2MASS Atlas, plus other material from CFHT and OHP. The extended outer wings of the PSF were measured and their effect on such features as axis-ratio and colours carefully checked. In our data, the red-halo CCD phenomenon is the unique cause of a bluer major-axis in $V-I$. Morphological indices suggest that the disk of NGC 3115 was once a complete little spiral, before being "swallowed" by a much bigger object. All colour distributions show the major-axis slightly redder than the minor-axis regions, at least not far from centre, in the radial range where the disk stands out. This is interpreted in the framework of a bulge+disk model as a metallicity effect, the disk having locally a larger $[\mathrm{Fe} / \mathrm{H}]$ by some $0.16 \pm 0.04$.
\end{abstract}

Key words. galaxies: elliptical and lenticulars, $\mathrm{cD}$ - galaxies: ISM

\section{Introduction}

NGC 3115 is a well-studied object. At a distance of $8.83 \mathrm{Mpc}$ (Prugniel \& Simien 1996) its halo red giants have been resolved (Schulte-Ladbeck et al. 2003), while many globular clusters could be observed in detail (Kuntschner et al. 2002; Puzia et al. 2002). In the Color Atlas of Galaxies, Wray (1988) describes NGC 3115 as an "edge-on highly evolved system in which we can see only old yellow stars". Many authors have tried to refine this perhaps approximate description by detailed measurements of either colours (Silva et al. 1989, or SBJ89), or more recently of spectroscopic indices (Bassin \& Bonatto 1978; Norris et al. 2006, or NSK06), in order to derive the ages, metallicities and the abundance ratio $[\alpha / \mathrm{Fe}]$. Since it seems appropriate to separate the object into bulge and disk components (Capaccioli et al. 1988; SBJ89; Scorza \& Bender 1995, or SB95; Michard 1998 or M98) the obvious option is to characterise distinct populations for these, and to infer possible evolution scenarii. One should add that the kinematics of NGC 3115 have been observed with high accuracy, for instance by Capaccioli et al. (1993), Fisher (1997), Emsellem et al. (1999), or NSK06. Fisher compares the results of several previous papers.

According to SBJ89, the disk population of NGC 3115 is 0.50 mag bluer in $B-i$ than its bulge, "a difference much larger than expected for a typical S0 galaxy" to quote the authors. The results of Michard \& Poulain (2000) in $B-R$ do not confirm this. It was later shown (Michard 2002, or M02) that the outer wings of the PSF may have important effects on the photometry of galaxies, the more so for flattened objects. In the $I$ band the CCD target often suffered from the red halo phenomenon, with dramatic errors induced on $I$ photometry and $V-I$ colour distributions. A model of NGC 3115 was shown to develop a blue disk in $V-I$ and a red disk in $U-V$ if convolved with the measured PSF in the OHP observations of 2000-1. These contradictions prompted us to reconsider the UBVRI colour distributions of this selected object, using new material and adding the
$V-J H K$ colours to be obtained from the 2MASS atlas (Jarrett et al. 2003).

In the following, Sect. 2 describes the observations that have been collected and the techniques used for their analysis. A strong emphasis is put on various systematic errors, notably those resulting from the red halo in the $I$ band and the often nonnegligible outer PSF wings in other bands. Section 3 deals with the morphology of NGC 3115. It introduces a two-component model, useful for the later discussion of the structure and population data. Section 4 is devoted to a description of colour measurements and the study of errors associated with outer PSF wings. In Sect. 5 an attempt is made to infer some properties of the stellar populations in NGC 3115.

\section{Technique and potential errors}

Several sets of images of NGC 3115 were available for the present discussion.

1. UBVRI frames obtained at the LCO (Las Campanas Observatory) with the 100 in. telescope in Dec. 1995 for $U V I$, Mar. 2001 for $B$ and Dec. 2001 for $R$. This material of superior quality forms the basis of the present work. Part of it has been applied by Kuchinski et al. (2000) to comparisons with their far-ultraviolet images. The frame resolution is $1^{\prime \prime}$ or slightly better, and the field reaches $8.9 \times 8.9^{\prime}$ (scale $0.26^{\prime \prime} /$ pixel). These precious images were made available through the generous help of Dr. Madore.

2. $B$ and $R$ frames were obtained in 1989 by the late J.L. Nieto at the Cassegrain focus of the CFHT (Canada-France-Hawaii Telescope) at a scale of $0.108^{\prime \prime} /$ pixel. Part of these are of subarcsecond resolution, but their field is limited to $1.15 \times 1.8^{\prime}$. This material has been used by Nieto et al. (1991a,b) or by Michard \& Poulain (2000).

3. UBVRI frames obtained with the $120 \mathrm{~cm}$ telescope of the OHP (Observatoire de Haute-Provence) in 1991 at a scale 
Table 1. Particulars of the used Las Campanas Observatory (LCO) frames of NGC 3115. Key to notes. SatA: saturation due to register overflow. SatB: saturation due to "burned out" central CCD columns.

\begin{tabular}{llllll}
\hline \hline Date & Filt & Exp & $F W H M$ & Sky & Note \\
\hline $95 / 12 / 25$ & $U$ & 300 & 1.12 & 21.95 & - \\
$95 / 12 / 25$ & $V$ & 300 & 0.85 & 21.75 & satA \\
$95 / 12 / 25$ & $I$ & 300 & 1.05 & 19.17 & satB \\
$01 / 03 / 18$ & $B$ & 1200 & 1.08 & 21.85 & satB \\
$01 / 12 / 21$ & $R$ & 30 & 1.06 & 20.94 & - \\
$01 / 12 / 21$ & $R$ & 300 & 1.06 & 21.13 & satB \\
\hline
\end{tabular}

of $0.84^{\prime \prime} /$ pixel. The resolution is around $3^{\prime \prime}$ in a field of $4.7 \times 7.5^{\prime}$. The $\mathrm{U}$ frame is of low $\mathrm{S} / \mathrm{N}$ and the $I$ frame saturated. This material has been studied in Michard \& Poulain (loc. cit.).

4. UBVRI frames from the same telescope taken in Jan. 2001 by J. de Freitas Pacheco and measured by Idiart et al. (2002) along with their sample of ellipticals. The field is $11.5^{\prime}$.squ. with a resolution worse than $3^{\prime \prime}$. The scale is $0.68^{\prime \prime} /$ pixel.

5. JHK images from the 2MASS Large Galaxies Atlas (Jarrett et al. 2003) with a resolution of about $3^{\prime \prime}$ and a $10^{\prime}$ field for a scale of $1^{\prime \prime} /$ pixel.

6. $B$ and $R$ images of the DSS (Digital Sky Survey) usable to check the morphology of the object at large radial distances, i.e. up to 8 arcmin along the major axis. The DSS images are saturated in a large inner radius range.

Detailed information about the CFHT and OHP frames has already been published. Table 1 gives full particulars for the LCO frames, notably the measured sky background and the $F W H M$ of their PSF.

\subsection{Analysis technique}

\subsubsection{Frame preparation}

This stage implies the mapping and substraction of the sky background, the cancellation of stars interfering with the galaxy image, a cosmetic treatment dealing with cosmic rays or other bad pixels, a photometric calibration from aperture photometry (Poulain 1988).

We added to these traditional operations a special treatment of the several centrally saturated frames from the LCO. Saturation may occur due to an insufficient capacity of the electronic registers, limited to 32768 counts, or in worse cases the incomplete transfer of strongly illuminated columns. In these instances, we "grafted" the central part of an unsaturated frame of equivalent quality upon the defective area. Thus the $300 \mathrm{~s}$ exposure $R$ frame received an insert from the $30 \mathrm{~s}$ exposure obtained in succession during the same night, which is a straightforward operation since a small shift derived from several stars allows a good co-centering, cross-correlation, and merging. In other cases, inserts from another telescope and/or colour had to be used: the LCO $B$ and $V$ images were grafted with $B$ and $R$ CFHT frames. The operation is then technically difficult and may leave imperfections in the innermost arcseconds of the resulting image.

\subsubsection{Isophotal analysis and image synthesis}

We routinely analyse the isophotal contours of available frames according to the precepts of Carter (1978), deriving the bestfitting ellipses at $0.1 \mathrm{mag}$ intervals. It is defined by its centre $x_{0}$, $y_{0}$, its major-axis $a$, of orientation PA, and the axis-ratio $q=c / a$. The isophotal radius is $r=(a c)^{1 / 2}$. The coefficients $c_{n}$ and $s_{n}$ of cosine and sine terms, respectively, in the harmonic expansion of deviations from this ellipse are also found. The value of $n$ reaches 10 in our routine: $c_{10}$ is necessary to describe the very pointed isophotes of NGC 3115.

A useful application of the data file containing the frame isophotal analysis is to reconstruct the image with such changes as wished: elliptical isophotes by putting all $c_{n}, s_{n}$ to zero; twoaxis symmetry with a fixed centre, an unique orientation, and even $c_{n}$ coefficients only; central symmetry if only even harmonic coefficient are kept. Then the comparison of such a reconstructed image with the original one helps to detect various peculiarities, such as asymmetries, dust patches, or the presence of faint objects.

\subsubsection{Colour measurements}

Our prefered technique for measuring colours is to average the relevant surface-brightness in well defined areas. These are located through a given set of isophotal contours, defining a convenient coordinate system applied (so to speak) on the two used frames. For instance, we used the system of isophotal contours in the LCO $V$ frame to recover the same test areas in all frames fron this series.

Azimuthal colour distributions are measured in one or several "rings" limited by isophotal contours. The eccentric anomaly $\omega$ in the average contour inside the ring is taken as abscissae, or the polar angle $\theta$ if desired. The relation between these angles is $\tan \theta=q \tan \omega$ : in NGC 3115 the axis-ratio is $q=0.35$ at $a=100^{\prime \prime}$.

Radial colour distributions are obtained from averaging in small domains limited by successive contours and a given range of $\omega$. At the isophotes with $q=0.35, \omega= \pm 15^{\circ}$ defines a "pencil" along one half of the major-axis, with an aperture $\theta= \pm 5.35^{\circ}$; the range $\omega=90 \pm 15^{\circ}$ gives a "fan" along the minor-axis with $\theta= \pm 52.6^{\circ}$. Integrating along the complete $\omega$ range gives the "isophotal" colour.

\subsubsection{The case of $V-J, V-K$ colours}

To measure colour distributions in these colours we have to equalise, as well as possible, the PSF in the corresponding passbands. Two methods were tried for this purpose:

- in the first one, an adjustment function was obtained by deconvolution of the broad 2MASS PSF by the narrow $V$-band LCO PSF, and then the $V$ LCO image was convolved by the adjustement function. Clearly this technique cannot give perfect results: the complex history of light pencils between the source and the 2MASS atlas (Skrutskie et al. 2006) cannot be rigorously described by a convolution,

- in the second approach, we first obtained, by deconvolution, a model $V$ image largely "cleaned" from the relatively modest effects of the LCO PSF. This model image was then rebinned and convolved with the 2MASS PSF to obtain an adequate $V$ image to be compared with the 2MASS frames.

These two techniques give $V-J$ and $V-K$ results in reasonable agreement. The derivation of the $J-K$ profiles is straightforward. 


\subsection{Well known and often neglected sources of errors}

\subsubsection{Seeing FWHM}

The effect of the inner PSF on various photometric quantities and on the colours has been studied by many authors (Schweizer 1981; Franx et al. 1989; Peletier et al. 1990; Michard 1999). In this later work, and also in Idiart et al. (2002), the usable radial range of colour measurements was extended by equalising the $F W H M$ of the involved frames, either by convolution or deconvolution. Such operations were not generally needed with the LCO frames, of remarkably similar FWHM: only the $U$ and $V$ frames $F W H M$ were "matched" for measuring $U-V$.

\subsubsection{The outer PSF wings}

Following the pioneering work of Capaccioli \& de Vaucouleurs (1983), the outer wings of the PSF, outside the region controlled by "seeing", were measured in 3 observing sessions at the OHP in 2000-1 (M02): these extensions were found up to a radius of circa $3^{\prime}$, and down to a level of $10^{-6}$ of the peak value (M02). The PSF wings are due to dust in the atmosphere and to microroughness of the main mirror and dust on its surface (Hassan $\&$ Burrows 1995). These features influence the photometry of the outer regions of galaxies, while colour measurements are seriously affected when they imply the comparison of two passbands with different PSF wings.

\subsubsection{The "red halo" in the I band}

Many thinned CCD are prone to an instrumental source of extended PSF wings known as the "red-halo", first described from laboratory experiments by Sirianni et al. (1998). It is due to multiple scattering in the CCD header, and could be much reduced by an appropriate modification. The residual effects in the Active Camera for Surveys are analysed by Sirianni et al. (2005). Due to the red-halo, the wings in the $I$ band are 2 to 3 times brighter than in the $B$ or $V$, at least in our OHP observations of 2000-01 with the Tek1024 CCD. The classical, small negative radial gradient of the $V-I$ colour in E-type galaxies (Bender \& Möllenhof 1987; Goudfrooj et al. 1994) becomes positive, and strongly so for not too large or flattened objects. Experiments in M02 with a model of NGC 3115 show a very blue disk in $V-I$ due to the red halo wings. These findings prompted us to measure the outer $P S F$ wings systematically for all the material used in the present study. A summary of the most useful results is given in Fig. 1.

The outer PSF wings of the LCO frames for the epochs and pass-bands of the NGC 3115 data were measured, using the stars of the NGC 3115 field, and of miscellaneous fields that happened to be in the material available to us. Due to the small number of suitable frames, we could not obtain precise results except in the $I$ band. Figure 1 (left panel) summarises our average PSF, leading to the following remarks:

- The PSF outer wings are the same in $B, V, R$ within errors.

- The red halo is very important, as the PSF wings are 10 times brighter in $I$ than in $B V R$ at a radius of about $10^{\prime \prime}$. A visual comparison of bright stars images in $I$ and another colour is sufficient to see the red halo on these frames.

- The outer wings in $U$ are found brighter than in $B V R$. Note that only two discordant measurements were available in this band.

- The PSF wings are much less extended at the LCO than at the OHP, probably due to the less dense and much cleaner

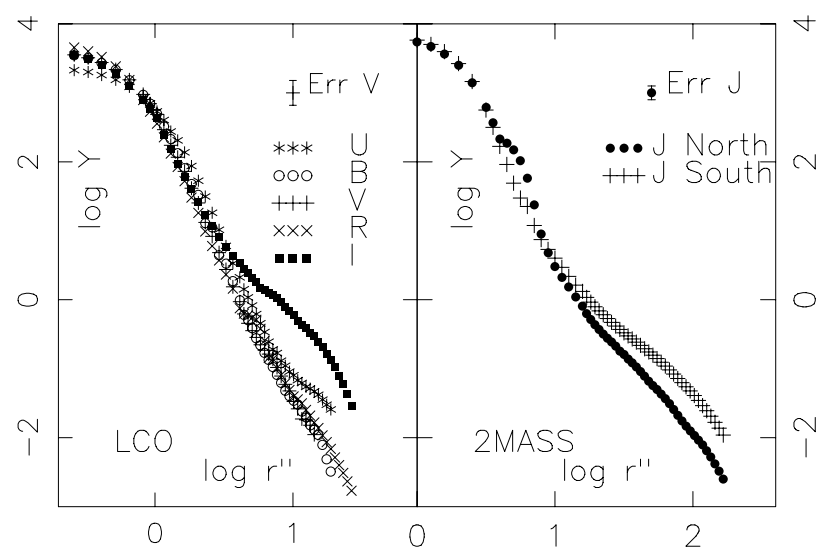

Fig. 1. Profiles of the PSF of LCO frames (left panel) in UBVRI and 2MASS frames (right panel) in $J$ for both used telescopes. Abscissae: $\log r^{\prime \prime}$. Ordinates: surface brightness $\log Y$ in arbitrary units. Among the LCO PSF note the intense red halo in the $I$ band, while the others are quite similar. In 2MASS the JHK PSF are the same within errors but the wings are brighter for the southern instrument.

mountain atmosphere. In $B V R$ the $10^{-6}$ level is attained at about $23^{\prime \prime}$ rather than at $3^{\prime}$ !

The PSF far wings of the 2MASS images were obtained separately for the two telescopes at Cerro Tololo and Mount Hopkins, for the southern and the northern data respectively (Fig. 1, right panel). We used fields of open clusters to measure unsaturated stars plus stars of moderate saturation, completed with fields containing bright stars with extended instrumental aureoles. The PSF far wings for the 2MASS images are rather bright, the more so for the southern instrument. They appear nearly the same in $J, H, K$.

As regards the OHP observations, the PSF far wings in 2000-1, where a CCD Tek1024 was in use, were described in M02. Those of our 1991 run, with an RCA CCD, could be measured in BVRI only from one field. They were found to differ from the 2001 results, fainter in all pass-bands, notably in $B$. The red-halo in the $I$ band was also present but much less pronounced than with the Tectronics target later in use.

\subsubsection{Sky background errors}

Modest errors in the evaluation of the average sky background produce significant errors in galaxy photometry at low light levels and prohibitive errors in colour estimates. In the case of E-type objects, a background error for one frame, or for both, involved in the measurement of a radial colour gradient introduces a characteristic break in the linear run of colour against $\log r$. This unwanted feature may be suppressed by correcting one of the background estimates, as in Michard (2000). In the present work, we matched the background of all used frames to the one of the LCO $V$ frame (kept unchanged) in order to extend the linear range of relations between isophotal colours $V-C$ and $\log r, C$ being any colour from any of the series. The needed "corrections" give crude statistics of relative background errors, i.e. expressed in $\%$ of the measured background. The sample for 11 estimates has a mean of $0.4 \%$ with $\sigma=0.8$. The sky background is more often overestimated than the reverse, that is some truncation of the outer galaxy is introduced.

Besides errors in the adopted average sky background, there may exist local large scale variations in this background, due to imperfections in the "flat-field" of the frame. This may occur 


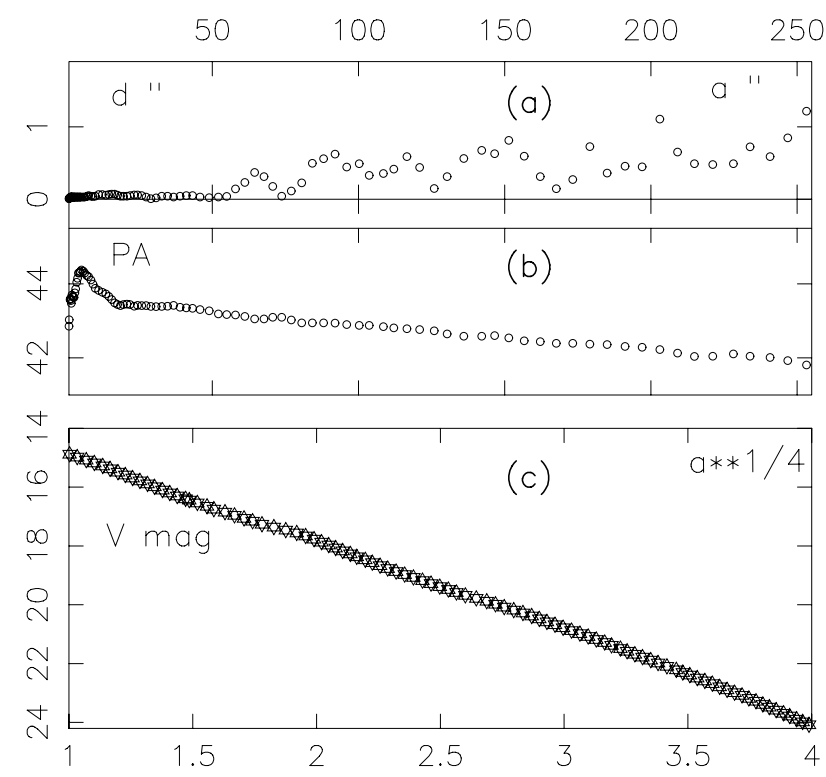

Fig. 2. Isophotal analysis of the LCO V image of NGC 3115. Upper frames a) and b) with the isophotal major-axis $a^{\prime \prime}$ in abscissae. Ordinates: a) the excursions $d^{\prime \prime}$ of the centre of isophotes. b) the position angle PA of their major-axis. Lower frame with $a^{1 / 4}$ in abscissae. In ordinates: c) surface brightness in $V$ mag.

because the spectrum of the flat-field source is different from the spectrum of the night-sky. We believe that some background large-scale fluctuations remain after the usual corrections.

\section{Morphology of NGC 3115}

\subsection{Observational results from LCO frames}

According to Michard \& Marchal (1993), NGC 3115 contains a disk of nearly edge-on inclination (SB95) fully embedded in a spheroidal halo. In SB95 the D/B ratio is found to be 0.11 .

1. Surface brightness distributions. Plots of the $\mathrm{SuBr}$ distribution against the major-axis $a^{1 / 4}$ (see Fig. 2) indicate remarkable agreement with the classical $r^{1 / 4}$ law. This diagram does not show the "hump" above the $r^{1 / 4}$ law often found in S0, such as NGC 4350, 4570, and others. If projected nearly face-on, the disk would perhaps disappear, and NGC 3115 would be classified as elliptical (see also Capaccioli et al. 1991).

2. A highly symmetric object. A primary test of the symmetry of NGC 3115 is made from the positions of the apparent centre of the isophotes. In the measurements of the LCO $V$ frame (see Fig. 2) this position does not deviate by more than $1^{\prime \prime}$ from the actual centre: this applies to the total measured range of $a=250^{\prime \prime}$ or 9.68 mag. Larger deviations are found for poorly exposed CCD frames or for the DSS plates. Another symmetry test is obtained from the $c_{3}$, $s_{3}$ coefficients, which remain below 0.004 at a local "peak" and have the same sign, involving opposite effects. It appears than NGC 3115 is a remarkably symmetric galaxy in the range covered by the LCO $V$ frame. Photometric errors are not likely to introduce symmetry where absent!

3. "Twist of the isophotes". The isophotal analysis of all the frames shows a small but well defined "twist" of the contours, with a PA variation remarkably linear in terms of the major-axis $a$ (see Fig. 2). The PA variations are found to be

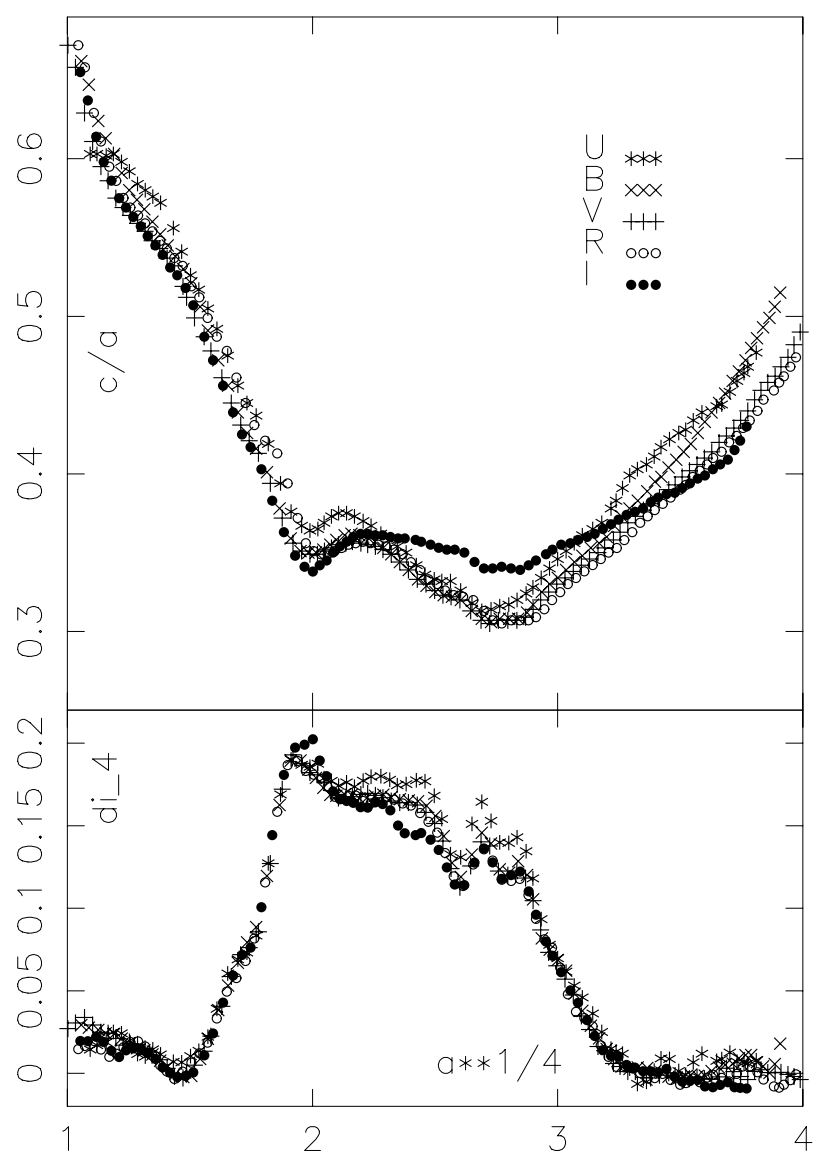

Fig. 3. Comparison of isophotal parameters in various colours. Abscissae: $a^{1 / 4}$ in ". Upper panel: radial profiles of the axis-ratio $q$ for the 5 LCO images. Lower panel: radial profiles of the disk contrast $d i_{4}$ for the $5 \mathrm{LCO}$ images. See comments in the text.

the same in the various colours and in different sets of observations. The absence of curvature and the constancy of the slope up to more than twice the extent of the disk (see below), indicate that the twist is a property of the bulge-halo component, which is probably slightly triaxial (de Zeeuw \& Franx 1991).

4. Axis-ratios. Figure 3 shows radial profiles of the $q$ axis ratio for the $5 \mathrm{LCO}$ frames. The $I$ band curve far deviates from the others, due to pollution by the red-halo. The $B, V, R$ curves are nearly coincident in the inner regions $\left(a<80^{\prime \prime}\right)$, but the $B$ profile deviates at larger $a$, with q increasing well above the $V$ and $R$ values. The $U$ band curve deviates at all radii, in part due to a difference in seeing, to a more uncertain photometry, and perhaps to a colour effect associated with the disk.

This colour effect in the $q$ ratio occurs neither in DSS frames, nor in our OHP frames (otherwise spoilt by strong PSF wings), and no convincing explanation can be offered. We therefore have to consider a priori the colours involving the $U$ and $B$ bands as uncertain for $a>80^{\prime \prime}$.

5. The cosine coefficients $c_{4}, c_{6} \ldots$ contribute to define the true major-axis $l$ of an isophote. as compared to the one of the best-fit ellipse $a$, with $l=a\left(1+c_{4}+c_{6}+c_{8}+c_{10}\right)$. Since they remain well correlated, we have introduced an index of diskyness as $d i_{4}=c_{4}+c_{6}+c_{8}+c_{10}$, or $l=a\left(1+d i_{4}\right)$. This index is plotted in Fig. 3 for the $U B V R I$ LCO frames. It falls to zero in all colours, as do all terms in its definition, near $a=110^{\prime \prime}$. This shows that the disk vanishes at this major-axis value. An inner maximum of $c_{4}$ near $a=1.5^{\prime \prime}$ was noted by Nieto 


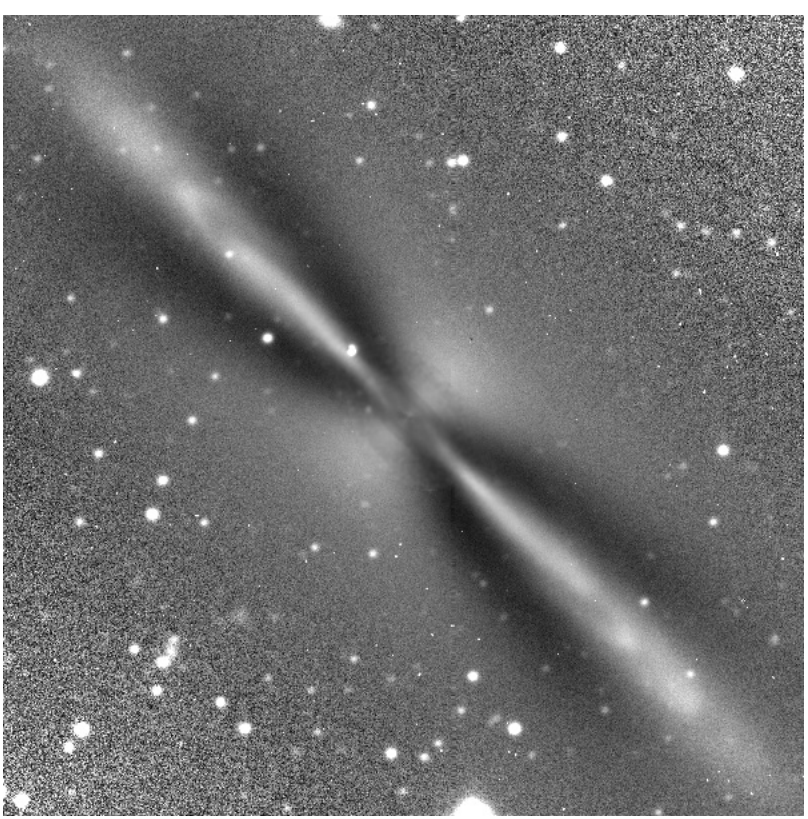

Fig. 4. Differential image built from the LCO $B$ frame. A dummy with elliptical isophotes has been substracted, in magnitudes, from the observed frame. Note the crest of the disk with nearly symmetrical breaks and secondary maxima. Side of the field: $156^{\prime \prime}$ or $6.55 \mathrm{kpc}$.

et al. (1991); it can be better seen in the CFHT images than in Fig. 3. The disk contrast may have a secondary peak at this position and may vanish close to the galaxy centre. Only the $U d i_{4}$ profile differs slightly fron the curve common to the $B V R$ pass-bands. It appears that the bright PSF wings in the $I$ band have only minor effects on the $d i_{4}$ parameter.

6. Dust or fossil spiral structure in the disk? By substracting a model with elliptical isophotes from the observed image (see Sect. 2.2.2) we obtain a difference image emphasizing the disk contrast against the mixed bulge. Figure 4 gives an example of such an exercise, made with the $V$ LCO frame. One may see 4 "peaks" in the disk ridge-line, 2 at the SE and 2 at the NW at practically symmetrical positions, separated by 4 "dips". We find radial distances of 60.8 and 75.4 " for the peaks.

Following Michard \& Simien (1993) we have looked for the "minor-axis asymmetry", a feature very sensitive to the occurence of a layer of dust inside the disk, and so prominent in edge-on spirals. The high resolution material of CFHT and LCO show no evidence of significant asymmetry along the minor-axis, i.e. no dust can be detected.

\subsection{A disk+bulge model of NGC 3115}

We have tried to improve previous models based on the morphological parameters (SB95, M98) using the LCO $R$ frame, with the advantages of good seeing, moderate outer PSF wings, and large field. In our model both bulge and disk have elliptical isophotes. There are no a priori constraints on possible variations of the $q$ values or on the photometric profiles. Figure 5 gives profiles of the $R$ magnitude and $c / a$ ratios against the major-axis. Note the "plateau" in the $q$ ratio of the bulge, the outer cut-off of the disk, the limited range of $a$ where the disk brightness exceeds the one of the bulge. An isophotal map of the two components is shown in Fig. 6. The model is calculated in a field of $a=468^{\prime \prime}$ by $c=234^{\prime \prime}$. In this range the ratio of the model

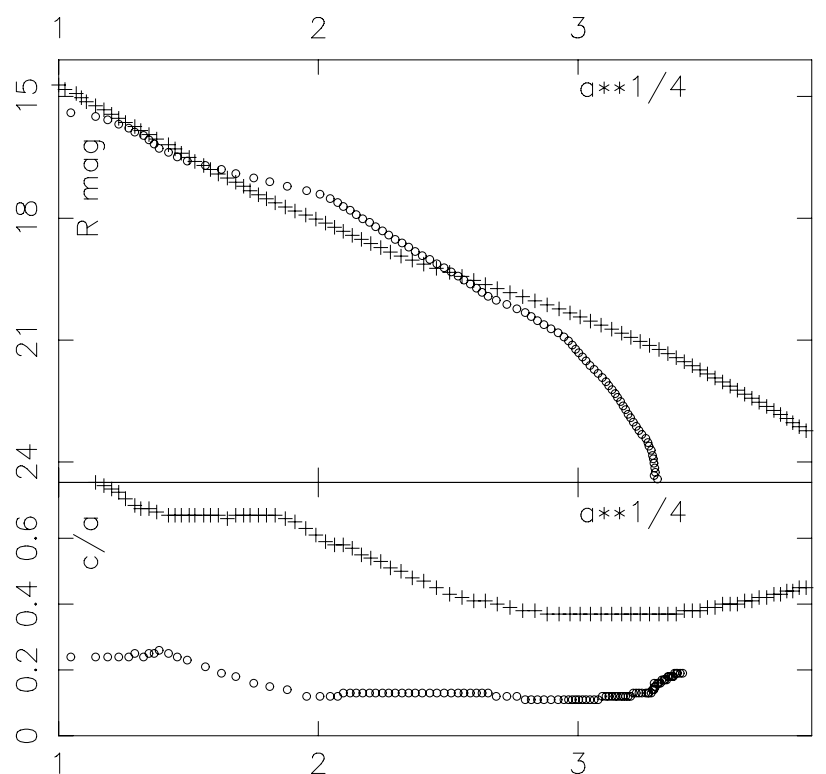

Fig. 5. Disk/bulge model of NGC 3115. Abscissae: $a^{1 / 4}$ in ". Ordinates: upper frame: bulge (crosses) and disk (open circles) $R$ surface brightness. Lower frame: bulge (crosses) and disk(open circles) $c / a$ ratios.

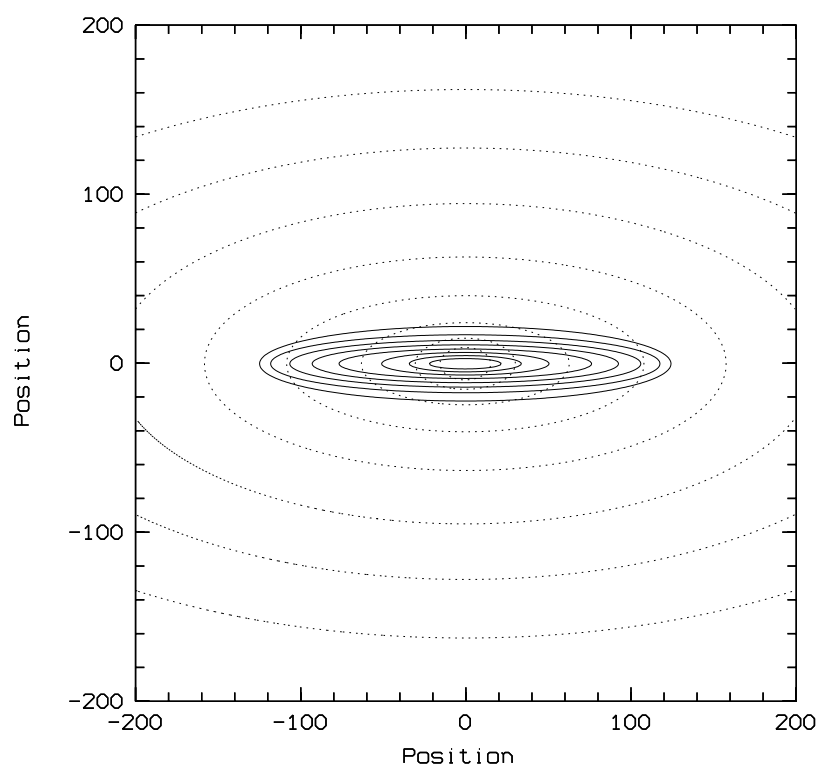

Fig. 6. A disk+bulge model of NGC 3115 with elliptical isophotes for both components. It represents a symmetrized version of the $R$ LCO image. The map shows isophotes at $R$ magnitudes 18 to 25 . The position scales are in $1^{\prime \prime}$ units. The side of the displayed field is $17.1 \mathrm{kpc}$.

image to the coresponding observed one has an average of 0.993 with $\sigma=0.023$ and extremal errors of \pm 0.05 . Since the plotted profiles are not constrained in our model, it may be significant that the disk $R$ magnitude is approximately linear in $a$. As seen in Fig. 6, the disk $c / a$ is constant in a large radial range, but increases near the feature centre: if real this might be the remnant of a fossil bulge. Added to the features described above (Fig. 4) this suggests that the present "disk" of NGC 3115 was a complete little spiral before being swallowed by a much larger object. 
Table 2. Isophotal colours at $r_{\mathrm{e}} / 2$ and gradients. Columns: (1) source, i.e. observatory and period; (2) colour; (3) range of radii in "; (4) zero-point colour at $r_{\mathrm{e}} / 2$ corrected for galactic extinction and $K$ effect; (5) Logarithmic gradient of the C colour $\Delta_{\mathrm{CC}}$.

\begin{tabular}{lllll}
\hline \hline$(1)$ & $(2)$ & $(3)$ & $(4)$ & $(5)$ \\
Sources & Colour & Radii & C-C & $\Delta_{\mathrm{CC}}$ \\
\hline LCO 95 & $U-V$ & $3-140$ & 1.487 & -0.182 \\
LCO 95-01 & $B-V$ & $3-100$ & 0.935 & -0.064 \\
LCO 95-01 & $B-R$ & $3-100$ & 1.500 & -0.112 \\
LCO 95-01 & $V-R 1$ & $3-100$ & 0.581 & -0.015 \\
LCO 95-01 & $V-R$ & $3-100$ & 0.566 & -0.048 \\
OHP 91 & $U-V$ & $4-40$ & 1.481 & -0.215 \\
OHP 91 & $B-V$ & $8-80$ & 0.953 & -0.059 \\
OHP 91 & $B-R$ & $5-80$ & 1.528 & -0.067 \\
OHP 91 & $V-R$ & $4-80$ & 0.575 & -0.008 \\
OHP 01 & $U-V$ & $5-100$ & 1.455 & -0.238 \\
OHP 01 & $B-V$ & $5-100$ & 0.925 & -0.069 \\
OHP 01 & $B-R$ & $5-100$ & 1.511 & -0.079 \\
OHP 01 & $V-R$ & $5-100$ & 0.590 & -0.010 \\
OHP 01 & $V-I$ & $6-80$ & 1.222 & -0.047 \\
2MASS & $J-H$ & $4-80$ & 0.672 & -0.041 \\
2MASS & $J-K$ & $3-80$ & 0.903 & -0.070 \\
LCO+2MASS & $V-J$ & $5-80$ & 2.448 & -0.098 \\
LCO+2MASS & $V-K$ & $4-80$ & 3.340 & -0.155 \\
OHP91+2MASS & $V-J$ & $9-90$ & 2.390 & -0.048 \\
OHP91+2MASS & $V-K$ & $9-90$ & 3.300 & -0.113 \\
\hline
\end{tabular}

\section{Colour distributions in NGC 3115}

\subsection{Results}

\subsubsection{Isophotal colours and gradients}

The results of linear fits of isophotal colours against $\log r$, noted $\Delta_{U V}$ (and similar for other colours), are given in Table 2. A semiradius $r_{\mathrm{e}} / 2=19^{\prime \prime}$ is adopted for the zero-point. Two divergent values were obtained for the $V-R$ from LCO observations, R1 refering to a lightly exposed frame. For the same source, the $\Delta_{V I}$ gradients are strongly affected by the red halo effects (see Fig. 9) and have been discarded. We also rejected $\Delta_{V I}$ from the OHP1991 data. The OHP2001 data are from Idiart et al. (2002) with the $V-I$ entries corrected for PSF wings effects by crossed convolutions of the $V$ frame by the $I$ PSF and conversely. To obtain unbiased gradients for $\Delta_{V J}$ and $\Delta_{V K}$ in the presence of very different PSF, we have treated the $V$ LCO frame to match its PSF to those of the 2MASS $J$ and $K$ frames (see Sect. 2.2.4). The gradients from the various obervations are in reasonable agreement. The $\sigma$ values of various sets of 3 measurements of the $\Delta_{U V}, \Delta_{B V}$, and $\Delta_{V R}$ gradients range from 0.005 to 0.028 .

Note that the zero-point colours in Table 2 are corrected for galactic extinction and $K$ effect, the adopted total reddening being 0.030 in $B-V$. This is also the case of model colours in Table 3, but not in the various graphs where only local colour variations are of interest.

\subsubsection{A test for colour contrasts between the disk and the bulge}

According to the disk/bulge decompositions of SB95 and M98, the contrast of the disk against the bulge has a broad maximum roughly in the range $13<a<33^{\prime \prime}$. By tracing the azimuthal colour distributions in a ring bound by the isophotes at these $a$ limits, a straightforward indication of systematic colour differences between the two components is expected. Figure 7

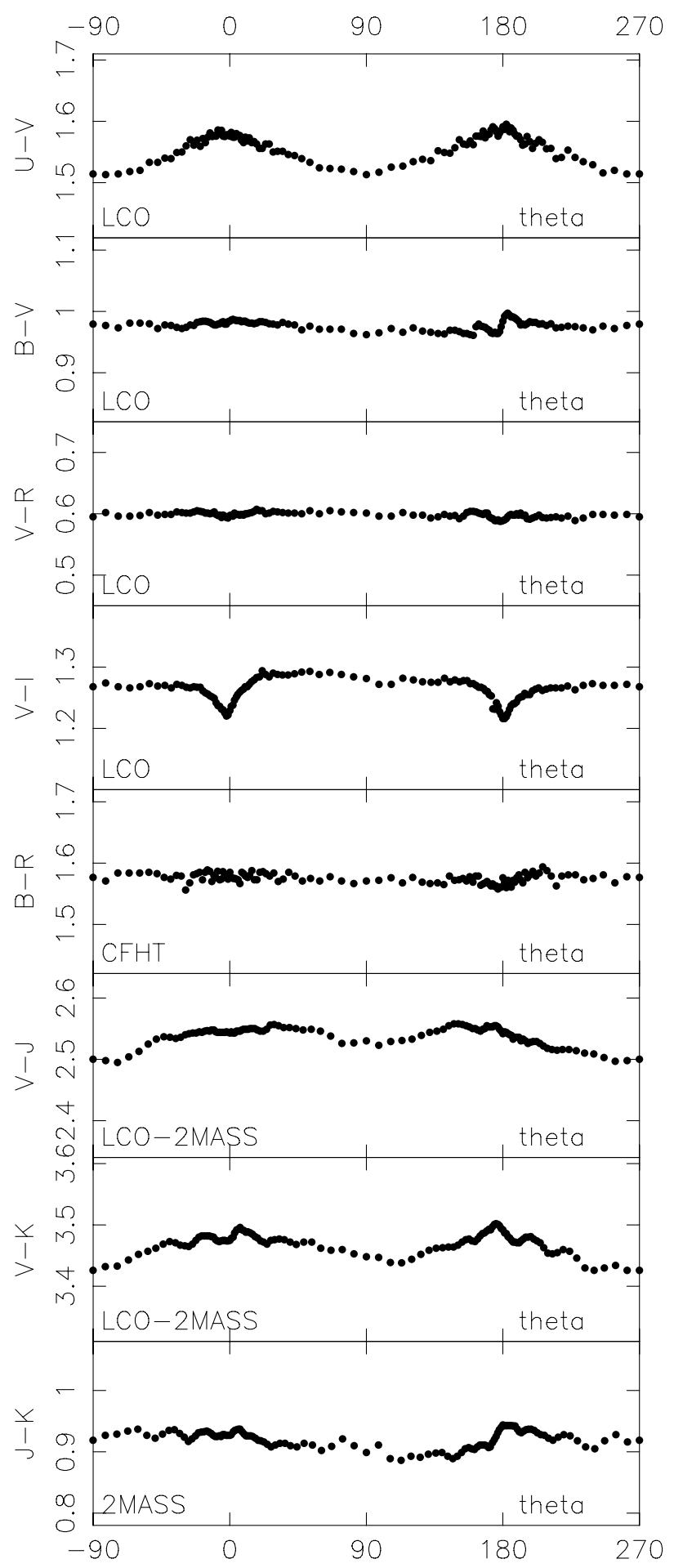

Fig. 7. Test of colour differences between bulge and disk from the azimuthal colour profiles in a ring limited by isophotes with $13<a<33^{\prime \prime}$. Abscissae: polar angle $\theta$ with major-axis at 0 and $180^{\circ}$. Ordinates: colours. The $V-I$ colour displays a spurious blue disk due to the redhalo of the CCD. The $U-V, V-J$ and $V-K$ profiles show evidence of reddening along the major-axis, this effect being negligible in $B-V$, $V-R, B-R$ and $J-K$.

displays the result of these experiments for all colours. The azimuthal colour diagrams are flat if disk and bulge have the same colour in the chosen $a$ range as is the case for $B-V, V-R$ and $B-R(\mathrm{CFHT})$. The $V-I$ colour displays a sharp "blue 
disk", an artefact as indicated below. The $U-V$ colour is redder near the major-axis by 0.06 at the disk position, i.e. $\theta=0$ or 180 degrees, and the $V-J, V-K$ colours show some evidence of the same behaviour. This reddeneing may be a consequence of the disk properties, i.e. stellar populations, although it appears negligible in the other colours (see a later discussion).

\subsubsection{Radial colour distribution along the major- and minor-axes}

The radial colour profiles have been measured separately for the major- and minor-axis, as indicated in Sect. 2.2.3, with the results shown in Fig. 8. For the high resolution data, LCO and CFHT, the inner range of the major-axis is redder than the minoraxis, and this reddening increases in amplitude and radial extent from $V-R$ to $U-V$. Farther out, the colour profiles along the two axis tend at first to merge. At large $a$ values, the colours in the two axes domains unfortunately behave in a rather erratic way, most probably due to photometric errors also showing in the $c / a$ profiles (see Fig. 3 and Sect. 3.1.4). The $V-J$, $V-K$ profiles have analogous properties: it seems however that the minor-axis profiles are depressed relative to the major-axis ones, and not the latter enhanced compared to the former. The operations needed to match the PSF of the LCO $V$ frame with those of 2MASS may introduce such anomalies.

Pending the discussion below, it appears that the new data is not compatible with the earlier finding of a blue disk. An intuitive explanation would imply a redder disk with an outwards blueing.

\subsection{Tests of colour data significance}

We have made various tests to ascertain the effect of the PSF on the above colour distributions, notably as regards the LCO frames data: an ad hoc "sharp" model, obtained from an observed frame by partial deconvolution, is convolved with the PSF from the various pass-bands and the resulting pseudo-colours distributions measured. Here are some results:

- False colours induced by the differences of the PSF among $L C O$ frames. The model is convolved with the 3 distincts LCO PSF, i.e. $U$, the average in $B V R$, and $I$. The false colours thus induced are very small in $U-V$ but dramatically large in $V-I$, as shown by Fig. 9, which should be compared with the actual measurements of Figs. 7 and 8: the prominent "blue disk" in $V-I$ seen in azimutal and radial colour distributions, is generated by the I PSF wings due to the redhalo phenomenon.

- False colours induced by measured PSF in OHP observations. It appears in this case that the wings of the respective $P S F$ in a pair of frames have full control over the occurrence of a red or blue disk according to our test of the azimuthal colour profile, or more generally of colour differences between the major- and minor-axis.

- Comparisons between OHP and LCO data. Some effort has been made to degrade LCO frames in $U B V R$ in order to match the PSF of the OHP2001 series, including their extended wings. The technique of this exercise is the first one described in Sect. 2.2.4. The outcome seems satisfactory, as the $q$ and $d i_{4}$ parameters measured for the OHP frames are well reproduced. The application is then to use the OHP frames, of large field and fair exposures, to test the photometry of the LCO frames in their outer regions. This casts further doubts on the $q$ variations in $B$ (and $U$ ?) at radii $a>80^{\prime \prime}$ already noted in Sect. 3.1.4.

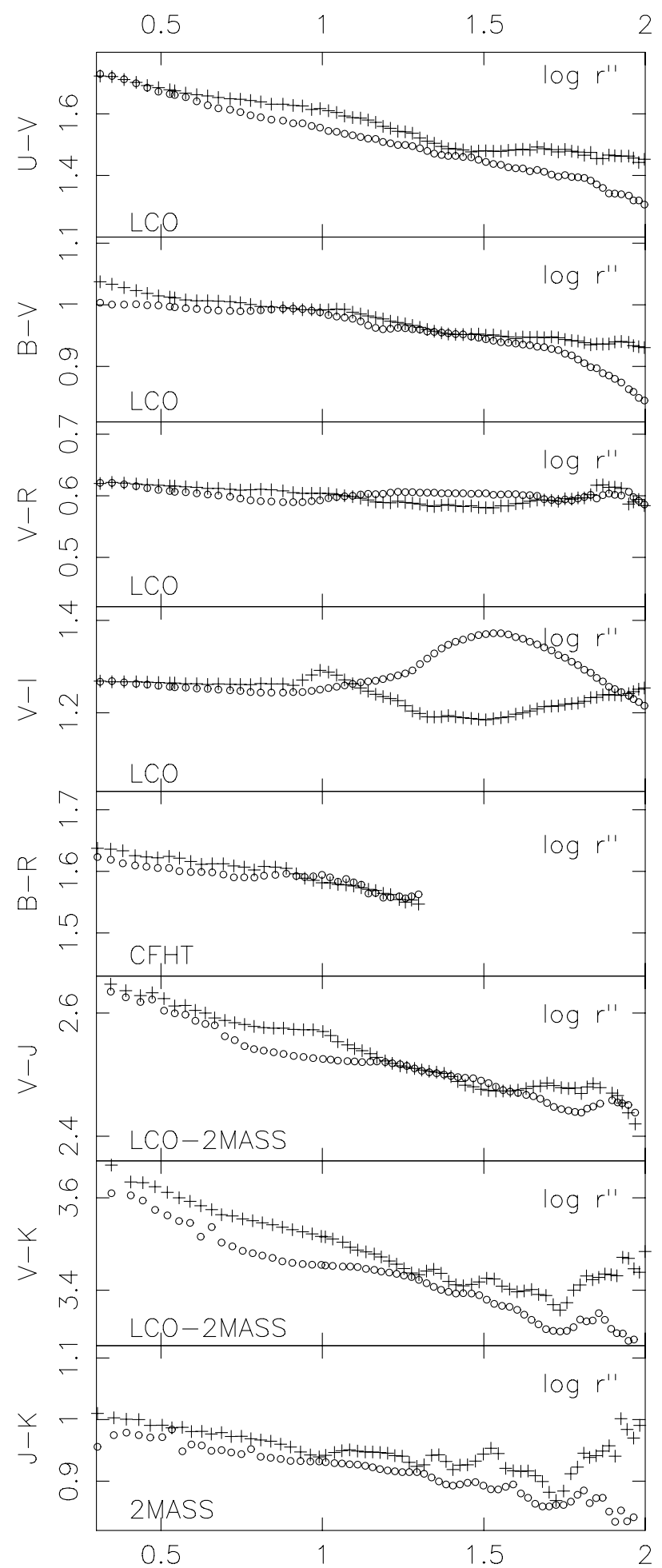

Fig. 8. Radial profiles of colours measured separately for the major-axis (crosses) and the minor one (open circles) along successive isophotes. Abscissae: isophotal radius $r$ in arcsec and logarithmic scale. Ordinates: colours. The large effect in $V-I$ is due to the red-halo in the $I$ pass-band. For other colours the major-axis is slightly redder than the minor-axis in the inner radial range.

\section{A model and derived variations in stellar populations}

\subsection{Model colour distributions}

Starting from the two-component model in red light of Sect. 3.2, we have obtained many models in the various pass-bands, 

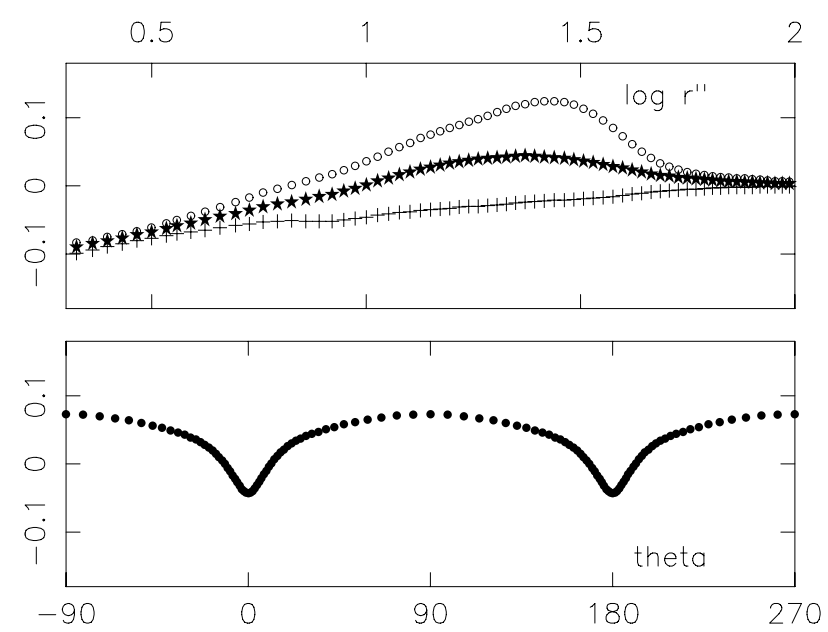

Fig. 9. Effects of the CCD "red-halo" on the $V-I$ colour distributions for the LCO frames. An ad hoc sharpened model is convolved with the measured PSF in $V$ and $I$, and the frames so degraded are used to measure the pseudo-colour distributions. The upper panel contains the radial distributions: besides the major-axis and minor-axis colours noted as in Fig. 8, it also give the isophotal colour (stars). The lower panel shows the azimuthal distribution in the usual range $13<a<33^{\prime \prime}$ already used in Fig. 7.

Table 3. Parameters of model colour-profiles and quality figures derived from the $\mathrm{O}-\mathrm{C}$ of colours in test domains. (1) Colour $C$. (2) Peak bulge colour $C \mathrm{bu}_{0}$ at $r<1^{\prime \prime}$. (3) Bulge gradients $\Delta_{\mathrm{bu}}=\mathrm{d} C / \mathrm{d}(\log r)$ adapted from Table 3 values for the whole galaxy. (4) Peak disk colour $C \mathrm{di}_{0}$ at $a<2^{\prime \prime}$. (5) Disk gradients $\Delta_{\mathrm{di}}=\mathrm{d} C \mathrm{di} / \mathrm{d} a$. (6) Standard deviation $\sigma_{m}$ of O-C residuals of $m C_{i}$ values representative of the mean colour radial profile, averaged on the two axis. (7) Standard deviation $\sigma_{\mathrm{d}}$ of $\mathrm{O}-\mathrm{C}$ residuals of $\mathrm{d} C_{i}$ values representative of the difference between major and minor-axes profiles.

\begin{tabular}{lllllll}
\hline \hline$(1)$ & $(2)$ & $(3)$ & $(4)$ & $(5)$ & $(6)$ & $(7)$ \\
$C$ & $C \mathrm{bu}_{0}$ & $\Delta_{\mathrm{bu}}$ & $C \mathrm{di}_{0}$ & $\Delta_{\mathrm{di}}$ & $\sigma_{m}$ & $\sigma_{\mathrm{d}}$ \\
\hline$B-V$ & 1.012 & -0.067 & 1.030 & -0.0018 & 0.007 & 0.010 \\
$V-R$ & 0.596 & -0.018 & 0.611 & -0.0008 & 0.004 & 0.011 \\
$B-R$ & 1.599 & -0.087 & 1.631 & -0.0030 & 0.005 & 0.012 \\
$U-V$ & 1.682 & -0.190 & 1.724 & -0.0035 & 0.008 & 0.009 \\
$V-J$ & 2.553 & -0.098 & 2.618 & -0.0035 & 0.010 & 0.009 \\
$V-K$ & 3.519 & -0.152 & 3.620 & -0.0045 & 0.018 & 0.022 \\
$J-K$ & 0.961 & -0.055 & 0.996 & -0.0015 & 0.007 & 0.015 \\
\hline
\end{tabular}

imposing various laws of colour variations separately for the bulge and disk components, and looking for the combinations of parameters which could restore reasonably well the observed data from the LCO and 2MASS series. Here are some technical details about the tested distributions:

- The bulge colour $C_{\mathrm{bu}}$ is constant for $r<1^{\prime \prime}$, then decreases linearly with $\log r$, as currently observed for E galaxies or for NGC 3115 itself. The slopes $\mathrm{d} C_{\mathrm{bu}} / \mathrm{d} \log r$ and the zero-points are often kept equal to the measured values of Table 3 for the galaxy as a whole, or eventually are slightly modified.

- The disk colour $C_{\mathrm{di}}$ is constant for $a<2^{\prime \prime}$, then decreases according to a specified law. We have tested 3 possible such laws: one simply linear in $a$, one linear in $\log a$, and a somewhat intermediate profile linear in $a^{1 / 2}$. The first one, i.e. a disk colour linear in $a$, tends to give better agreement with the observations. Also this particular profile is favoured, in early-type disk galaxies, by the extensive observations of de Jong \& van der Kruit (1994); see also de Jong (1996). It was thus selected for the final modeling.
- Model colour distributions were obtained for $B-V, B-R$, $V-R, U-V, V-J, V-K$, and $J-K$ for comparison with the data in Sect. 4.1. The parameter space was not exhaustively explored, but we were content with a limited number of experiments usually 4 to 10 in each colour. The first step in estimating the usefulness of a solution was to compare the graph of the calculated radial colour distribution with the graphs of Fig. 8. Then the colours of 20 test-areas were measured for the models as for the observed frames. These are limited by isophotal contours and arcs of the eccentric anomaly $\omega$ of elliptical isophotes, chosen as explained in Sect. 2.3.2. to describe the major and minor-axes domains. From the measurements the quantities $m C_{i}$ and $\mathrm{d} C_{i}$ were derived, describing respectively the mean colour profile along both axes and the difference between major and minor-axes. Finally the figures of merit of a model are the average and standard deviations of the Observed-Calculated $m C_{i}$ and $\mathrm{d} C_{i}$ values. Obviously the average $\mathrm{O}-\mathrm{C}$ should be near zero, and the standard deviations noted $\sigma_{m}$ and $\sigma_{\mathrm{d}}$ then expresses the ability of the model to represent the two profiles. The outermost test areas were discarded from the $\mathrm{O}-\mathrm{C}$ due to photometric uncertainties already noted.

The parameters of the adopted model colour-profiles and the above defined quality figures are collected in Table 4. It appears that $\sigma_{m}$ and $\sigma_{\mathrm{d}}$ are near 0.01 or smaller, except for the $V-K$ model where they reach 0.02 .

\subsection{Higher disk metallicity?}

In Fig. 10 we show the isophotal colour profiles of the bulge and disk of NGC 3115 according to the parametric representation of the models in Table 4. These results call for the following comments:

- The disk is distinctly redder in all colours in the internal part of the object, i.e. $a<50 \pm 10^{\prime \prime}$, then becomes as blue (or bluer?). The disk reddening remains nearly constant in the range $6<a<25^{\prime \prime}$ with a maximum near $a=12^{\prime \prime}$.

- As expected, the disk colour profiles depend heavily on the analytical form adopted for their representation, here a linear run against the major-axis $a$. Solutions with other assumed forms give similar disk colours in the radial range where the disk contributes the most to the total light. In the inner and outer ranges, the model profiles are only extrapolations of this significant range.

- It is tempting to attribute the redder disk colour to increased metallicity as compared to the bulge. Since the colour-metallicity gradients can be recovered from the theory of stellar population, a colour difference between bulge and disk readily provides a metallicity difference, neglecting possible age differences. Table 5 introduces the maximal colour excess of the disk models, estimated near $a=$ $12^{\prime \prime}$, the corresponding differences in $[\mathrm{Fe} / \mathrm{H}]$, and the differences in $\log Z$. The gradients $\mathrm{d} C /[\mathrm{Fe} / \mathrm{H}]$ are estimated from the SSP models at age 12 Gyr by Worthey (1994, W94). The gradients $\mathrm{d} C / \log Z$ are taken at the same age from the GALAXEV tables (Bruzual \& Charlot 2003, BC03). As expected, the maximum metallicity excess in the disk is found about the same for all colours. We find $[\mathrm{Fe} / \mathrm{H}]_{\mathrm{di}^{-}}$ $[\mathrm{Fe} / \mathrm{H}]_{\mathrm{bu}}=0.16 \pm 0.03$ with $\sigma=0.05$. On the other hand, $\log Z_{\mathrm{di}}-\log Z_{\mathrm{bu}}=0.23 \pm 0.06$ with $\sigma=0.9$ (or 0.20 with $\sigma=0.04$ if we reject the value for the $J-K$ colour). 


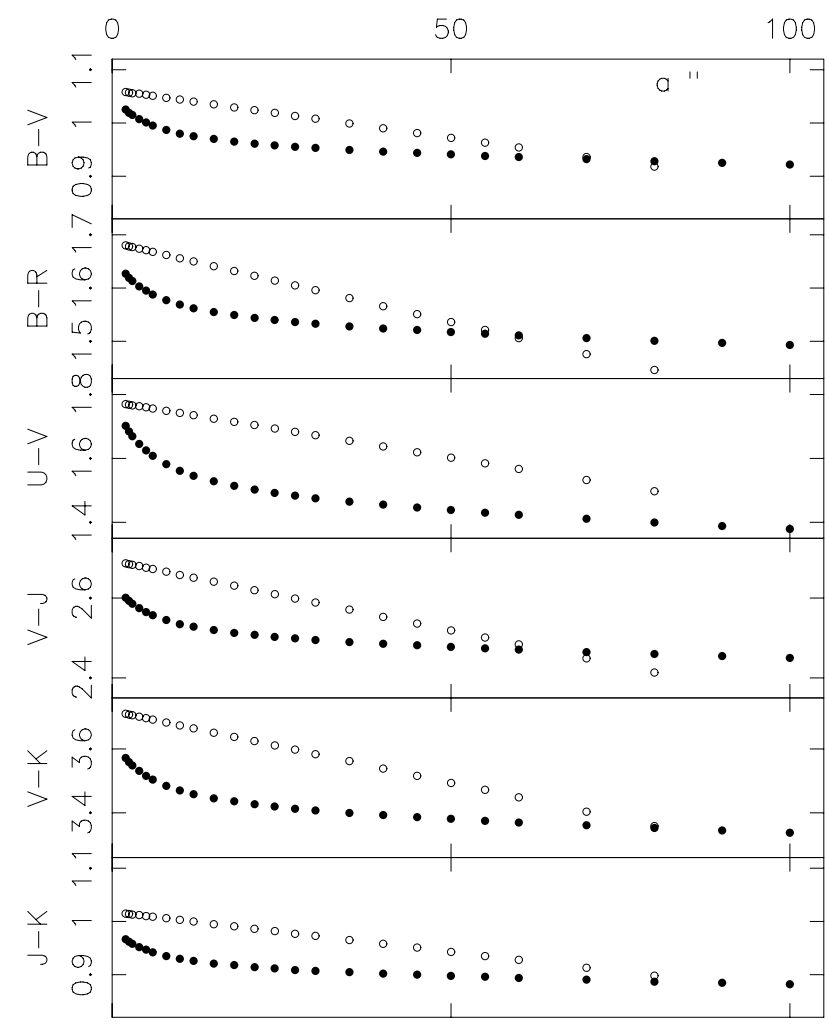

Fig. 10. Calculated colour distributions for the two components of NGC 3115 from the models derived in Sect. 5.1 with the parameters of Table 3. Bulge: full dots. Disk: open dots. The colour gradient of the disk is assumed linear in the major-axis $a$.

Table 4. Maximal disk reddening compared to bulge and derived metallicity excess. (1) Colour $C$. (2) Peak colour difference $C_{\mathrm{d}}-C_{\mathrm{b}}$ near $a=12^{\prime \prime}$ (3) Corresponding $[\mathrm{Fe} / \mathrm{H}]$ difference $[\mathrm{Fe} / \mathrm{H}]_{\mathrm{d}}-[\mathrm{Fe} / \mathrm{H}]_{\mathrm{b}}$ from calculations by Worthey (1994) (W94). (4) Corresponding $\log Z$ difference $\log Z_{\mathrm{d}}-\log Z_{\mathrm{b}}$ from calculations by Bruzual \& Charlot (2003) (BC03).

\begin{tabular}{llll}
\hline \hline$(1)$ & $(2)$ & $(3)$ & $(4)$ \\
$C$ & $C_{\mathrm{d}}-C_{\mathrm{b}}$ & {$[\mathrm{Fe} / \mathrm{H}]_{\mathrm{d}}-[\mathrm{Fe} / \mathrm{H}]_{\mathrm{b}}$} & $\log Z_{\mathrm{d}}-\log Z_{\mathrm{b}}$ \\
\hline$B-V$ & 0.065 & 0.18 & 0.25 \\
$V-R$ & 0.025 & 0.16 & 0.16 \\
$B-R$ & 0.088 & 0.18 & 0.21 \\
$U-V$ & 0.190 & 0.17 & 0.24 \\
$V-J$ & 0.123 & 0.09 & 0.14 \\
$V-K$ & 0.206 & 0.12 & 0.21 \\
$J-K$ & 0.074 & 0.25 & 0.42 \\
\hline
\end{tabular}

\subsection{Discussion}

- About colour data. The examination of Figs. 7 and 8 suggests an overall consistency in our data but with limited accuracy and possibly large errors in some colours and radial ranges. In the azimuthal colour profiles (Fig. 7), only the $U-V, V-J$, and $V-K$ profiles display a significant reddening near the azimuth of the major-axis, but a sharper peak in $\theta$ would be expected as the effect of a thin disk at small inclination. The radial profiles, on the other hand, (Fig. 8) show major-axis reddening in the inner range, which disappears outwards: see for instance the rather typical $V-J$ profile. The other profiles are qualitatively similar to this example, but we have to keep in mind the uncertainties in the effects of the PSF, chiefly in $V-J K$ since the restoration of a "common" PSF was a perilous exercise. In conclusion, the above modeling is consistent with all the data, but we cannot exclude the majoraxis reddening being smaller than obtained, and also the derived metallicity excess.

- Dust? A reddening of the disk could be due to an accumulation of dust. The colour excess would then be much larger in $V-J, V-K$ than in $U-V$, as calculated for instance by Buzzoni (1995): this is not observed. On the other hand, we found no evidence of dust from sensitive tests for dust patches or for the minor-axis asymmetry (see Sect. 3.1). For both these reasons we believe that dust does not play any role in the apparent reddening of the disk.

- Age differences? When extended to $V-J K$, the broad-band colours contain some separate information about the age $A$, as well as the metallicity $Z$ (or $[\mathrm{Fe} / \mathrm{H})$, of old stellar populations. This was noted by Buzzoni (loc. cit.) and remains true with other calculations of SSP models like the one of W94 or BC03 used above. A large enough age difference between disk and bulge would make the calculated metallicity excesses in $V-J K$ significantly different of their values in $B-V$ or other visual colours. This effect is not present in Table 4 suggesting the disk population to be roughly as old as the one of the bulge.

From comprehensive spectroscopic observations along both axes, NSK06 derive the radial profiles of several Lickindices, and estimate therefrom possible metallicity and age differences between the two regions. From their Fig. 5, I read a major-axis excess in $[\mathrm{Z} / \mathrm{H}]$ of circa 0.27 in a radial range near $a=20^{\prime \prime}$. On the other hand they find the populations in the major-axis domain (i.e. disk?) younger than in the minoraxis one (i.e. bulge?) by some 6 Gyr. If we combine these two results, we predict, from the W94 or the BC03 tables, that the colours along both axes should be nearly equal, the decrease of the age cancelling out the metallicity increase. This is not in agreement with our observations and their above interpretation.

- Remarks. There are however possible unconsistencies in NSK06 results (as in ours): it appears that only the $\mathrm{H} \beta$ index points to a younger major-axis, out of the 3 hydrogen lines measured. The major- and minor-axes profiles are the same in the $\mathrm{Mg}_{b}$ index, so that age and metallicity effects exactly cancel each other, a perhaps surprising coincidence. Finally, it would be difficult to identify the major-axis data with the properties of a small embedded disk like the one in Sect. 3.2 or in SB95. Disk and bulge are of about equal surface brightness on the major-axis, in the range $8<a<40^{\prime \prime}$, so that the apparent spectral differences are strongly attenuated in the emergent light, as compared to the actual spectra of each component. Then the true $[\mathrm{Z} / \mathrm{H}]$ excess inside the disk could be twice larger than estimated and exceed 0.5 , which appears unlikely. Also the disk vanishes near $a=100$ (or $r / r_{\mathrm{e}}=0$ in the paper under discussion), so that the $[\mathrm{Z} / \mathrm{H}]$ and age profiles of the two axes should merge before this limit.

\section{Concluding summary}

When studying from OHP frames (M02) the effects in galaxy photometry of the extended PSF wings, i.e. outside the seeing disk, the colour contrast of the major- against minor-axis of NGC 3115 was found to be essentially dependant of the PSF profiles, casting doubt on the often quoted result by Silva et al., of a very blue disk component in this galaxy. This prompted us to compile images of the object to try and get more convincing 
colour distributions. An essential point should be the complete knowledge of the PSF down to light levels of $10^{-6}$ of the peak value.

At our disposal were the Las Campanas frames of remarkably narrow PSF, except in the $I$ band affected by a strong "red halo". This was supplemented by the 2MASS Large Galaxy Atlas to provide the useful $V-J K$ and $J-K$ colours, and other material from CFHT and OHP already used in various other works (Sect. 2). The 2MASS PSF have very extended wings so that the derivation of $V-J$ or $V-K$ colours necessitates a special treatment to bring $V$ images to the appropriate PSF.

In Sect. 3 the morphology of NGC 3115 is studied from traditional isophotal analysis after Carter. Following earlier work in SB95 and M98, a bulge+disk model in the $R$ band is calculated (Sect. 3.2). The galaxy is an apparently dustless object, made up of:

- a large bulge-halo component of remarkable symmetry, grossly following the $r^{1 / 4}$ law, with a small isophotal twist suggestive of a slight triaxiality.

- a small embedded disk, vanishing near a major-axis $a=$ $100^{\prime \prime}$ or $4.3 \mathrm{kpc}$. Its ellipticity and surface brightness profiles are reminiscent of a small spiral, and it contains a "fossil structure" of nearly circular symmetry (also detected in NSK06).

The colour data are presented in Sect. 4. We find no evidence of a blue disk, except in $V-I$ where it is a proven artefact from the $I$ band red-halo. In all other colours the major-axis is reddened as compared to the minor-axis inside a radius of about $r<15 \pm 5^{\prime \prime}$ or $a \approx 20$ while the two profiles tend to merge farther out. Above $a \approx 60$ the profiles become erratic due to photometric errors.

Section 5 offers an interpretation of our colour observations in terms of a difference in metallicity between the bulge-halo and the embedded disk. Ad hoc colour changes are introduced in our bulge+disk model, and an acceptable agreement with observations is sought by trial and error. The reddening of the disk at its maximum corresponds to an $[\mathrm{Fe} / \mathrm{H}]$ increase of $0.16 \pm 0.04$, as calibrated by W94 SSP models, or $0.20 \pm 0.05$ in $[\mathrm{Z} / \mathrm{H}]$ from $\mathrm{BC} 03$ models, without large age differences. We finally discuss the compatibility of our results with recent line indices population studies.

Acknowledgements. We are deeply indebted to Dr. Madore who recovered the Las Campanas frames of NGC 3115 and auxiliary material from the archives of the Carnegie Institution and put them at our disposal: these are the basis of the present study.
Also used was material from the 2MASS Large Galaxy Atlas recovered from IPAC, from the CFHT telescope obtained by the late J.L. Nieto, from our observations at the Observatoire de Haute Provence with the late J. Marchal and with our co-workers T. Idiart et J. de Freitas Pacheco, from the Digital Sky Surveys. We are indebted to the referee, Dr. Buzzoni, for helpful suggestions.

\section{References}

Bassin, M., \& Bonatto, C. 2003, A\&A, 410, 803

Bender, R., \& Möllenhof, C. 1987, A\&A, 177, 71

Bruzual, G., \& Charlot, S. 2003, MNRAS, 344, 1000 (BC03)

Buzzoni, A. 1995, ApJS, 98, 69

Capaccioli, M., \& de Vaucouleurs, G. 1983, ApJS, 52, 465

Capaccioli, M., Vietri, M., Held, E. V., et al. 1991, ApJ, 371, 535

Capaccioli, M., Cappellaro, E., Held, E. V., et al. 1993, A\&A, 274, 69

Carter, D. 1978, MNRAS, 182, 79

de Jong, R. S., \& van der Kruit, P. C. 1994, A\&AS, 106, 451

de Jong, R. S. 1996, A\&A, 313, 377

de Zeeuw, T., \& Franx, M. 1991, ARA\&A, 29, 239

Emsellem, E., Dejonghe, H., \& Bacon, R. 1999, MNRAS, 303, 495

Fisher, D. 1997, AJ, 113, 950

Goudfrooij, P., Hansen, L., Jorgensen, H. E., et al., 1994, A\&AS, 104, 179

Idiart, T. P., Michard, R., \& de Freitas Pacheco 2002, A\&A, 383, 30

Jarrett, T. H., Chester, T., Cutri, R., et al. 2003, AJ, 125, 525

Kuchinski, L. E., Freedman, W. L., Madore, B. F., et al. 2000, ApJS, 131, 441

Kuntschner, H., Ziegler, B. L., Sharpless, R. M., et al. 2002, A\&A, 395, 761

Michard, R. 1998, A\&AS, 127, 299 (M98)

Michard, R. 1999, A\&AS, 137, 245

Michard, R. 2000, A\&A, 360, 85

Michard, R. 2002, A\&A, 384, 763 (M02)

Michard, R., \& Marchal, G. 1993, A\&AS, 98, 29

Michard, R., \& Poulain, P. 2000, A\&AS, 141, 1

Michard, R., \& Simien, F. 1993, A\&A, 274, L25

Nieto, J. L., Bender, R., Arnaud, J., et al. 1991a, A\&A, 244, L25

Nieto, J. L., Bender, R., \& Surma, P. 1991b, A\&A, 244, L37

Norris, M. A., Shatpless, R. M., \& Kuntschner, H. 2006, MNRAS, in press (NSK06)

Poulain, P. 1988, A\&AS, 72, 215

Puzia, T. H., Zepf, S. E., Kissler-Pattig, M., et al. 2002, A\&A, 391, 453

Schweizer, F. 1981, AJ, 86, 662

Schulte-Ladbeck, R. E., Drozdovsky, I. O., Belfort, M., et al. 2003, Ap\&SS, 284, 909

Scorza, C., \& Bender, R. 1995, A\&A, 293, 20 (SB95)

Silva, D. R., Boroson, T. B. T., \& Jedrzejewski, R. I. 1989, AJ, 94, 131 (SBJ89) Sirianni, M., Clampin, M., Hartig, G. F., et al. 1998, in Optical Astronomical Instrumentation, ed. S. d'Odorico, Proc. SPIE, 3355, 608

Sirianni, M., Jee, M. J., Benitez, N., et al. 2005, PASP, 117, 1049

Skrutskie, M. F., Cutri, R. M., Stenning, R., et al. 2006, AJ, 131, 1163

Worthey, G. 1994, ApJS, 95, 107 (W94)

Wray, J. D. 1988, The color atlas of galaxies (Cambridge Univ. Press) 